\title{
Two-dimensional modulational instability in photorefractive media
}

\author{
M Saffman $^{1}$, Glen McCarthy ${ }^{2}$ and Wieslaw Królikowski ${ }^{2}$ \\ ${ }^{1}$ Department of Physics, University of Wisconsin, 1150 University Avenue, Madison, \\ WI 53706, USA \\ ${ }^{2}$ Laser Physics Centre, Research School of Physical Sciences and Engineering, \\ Australian National University, Canberra ACT 0200, Australia
}

Received 17 November 2003, accepted for publication 14 April 2004

Published 4 May 2004

Online at stacks.iop.org/JOptB/6/S397

DOI: $10.1088 / 1464-4266 / 6 / 5 / 030$

\begin{abstract}
We study theoretically and experimentally the modulational instability of broad optical beams in photorefractive nonlinear media. The angular dependence of the growth rate of periodic perturbations is calculated. Our findings are confirmed by experimental measurements on a strontium barium niobate photorefractive crystal.
\end{abstract}

Keywords: photorefractive nonlinear optics, self-focusing, instabilities

A plane wave propagating in a medium with focusing nonlinearity is unstable with respect to the generation of small scale filaments [1]. This so-called modulational instability (MI) phenomenon has been extensively studied because of its importance as a factor limiting the propagation of high power beams. Filamentation may also be identified as the first stage in the development of turbulent fluctuations in the transverse profile of a laser beam [2]. In addition, MI is often considered as a precursor for the formation of spatial and/or temporal optical solitons. As far as optics is concerned, MI has been studied in media with various mechanisms of nonlinear response including cubic [1], quadratic [3], nonlocal [4, 5] and inertial $[6,7]$ types of nonlinearity. Importantly, MI is not restricted to nonlinear optics but has also been studied in many other nonlinear systems including fluids [8], plasmas [9] and matter waves [10].

In the context of optical beam propagation in nonlinear materials MI has usually been considered in media with spatially isotropic nonlinear properties. Recently a great deal of theoretical and experimental effort has been devoted to studies of nonlinear optical effects and soliton formation in photorefractive crystals [11-13]. While these media exhibit strong nonlinearity at very low optical power, their nonlinear response is inherently anisotropic [14]. The anisotropy causes a number of observable effects including astigmatic selffocusing of optical beams [15], elliptically shaped solitary solutions [16], geometry-sensitive interactions of solitons [17] and fixed optical pattern orientation [18].

Several previous studies of MI in the context of photorefractive media were limited to a one-dimensional geometry where the anisotropy is absent [19-21], and the physics is similar to the standard saturable nonlinearity [22]. On the other hand, in a real physical situation where one deals with finite sized beams, the anisotropic aspects of the photorefractive nonlinear response are expected to play a significant role. Some previous work [23, 24] already indicated the importance of anisotropy in the transverse breakup of broad beams propagating in biased photorefractive crystals. However, no detailed analysis of this phenomenon was carried out. In this paper we study the MI of optical beams in photorefractive media taking into account the full two-dimensional anisotropic model of the photorefractive nonlinearity.

Time independent propagation of an optical beam $\mathcal{E}(r, z)=(A / 2) \mathrm{e}^{\mathrm{i}(k z-\omega t)}+$ c.c. in a nonlinear medium with a weakly varying index of refraction is governed by the parabolic equation

$$
\frac{\partial A}{\partial z}-\frac{\mathrm{i}}{2 k} \nabla_{\perp}^{2} A(r, z)=\mathrm{i} k \frac{n_{2}(r, z)}{n_{0}} A(r, z) .
$$

Here $r=(x, y)$ and $z$ are transverse and axial coordinates, $\nabla_{\perp}=\hat{x}(\partial / \partial x)+\hat{y}(\partial / \partial y), k=2 \pi n_{0} / \lambda, \lambda$ is the wavelength in vacuum, $\omega=2 \pi c / \lambda, c$ is the speed of light and $n=$ $n_{0}+n_{2}(r, z)$ is the refractive index, with $n_{0}$ the spatially uniform background index and $n_{2}$ the spatially varying nonlinear increment.

In the case of a photorefractive screening nonlinearity the optical beam propagates through a photorefractive crystal externally biased with a DC electric field. The beam excites 
charges which, after migrating due to diffusion and drift in the applied field, are subsequently trapped by impurity or defect centres. The effective nonlinearity (refractive index change) is proportional to the low frequency electric field $\mathbf{E}$ created by light induced charge redistribution $[11,12,14]$. In the situation of interest here where the optical field is linearly polarized along $\hat{x}$ which coincides with the crystalline $\hat{c}$ axis, the nonlinear increment to the refractive index is given by $n_{2}(r, z)=-\frac{1}{2} n_{0}^{3} r_{33} E_{x}(r, z)$, with $r_{33}$ the relevant component of the electro-optic tensor and $E_{x}$ the $\hat{x}$ component of the low frequency electric field in the medium.

It is convenient to describe the nonlinear material response in terms of the quasi-static potential induced by the optical field. As shown in the appendix the resulting set of dimensionless equations is

$$
\begin{aligned}
& \frac{\partial A}{\partial z}-\mathrm{i} \nabla_{\perp}^{2} A=\mathrm{i} \frac{\partial \phi}{\partial x} A, \\
& \tau \frac{\partial}{\partial t}\left[\nabla_{\perp} \cdot\left(\hat{\epsilon}_{n} \nabla_{\perp} \phi\right)\right]+\nabla_{\perp}^{2} \phi+\nabla_{\perp} \phi \cdot \nabla_{\perp} \ln \bar{I} \\
& \quad=\frac{\partial}{\partial x} \ln \bar{I}+\frac{E_{\mathrm{ph}}}{E_{\mathrm{ext}} \bar{I}} \frac{\partial|A|^{2}}{\partial x}+\alpha \frac{\nabla_{\perp}^{2} \bar{I}}{\bar{I}}
\end{aligned}
$$

where $\bar{I}=\left(1+|A|^{2}\right) /\left[1-\xi \nabla_{\perp} \cdot\left(\hat{\epsilon}_{n} \nabla_{\perp} \phi\right)\right]$. The coordinates and variables have been normalized using the scalings given in the appendix with the addition of $|A|^{2} / \tilde{I} \rightarrow|A|^{2}$ with $\tilde{I}=2 I_{\mathrm{s}} /\left(\epsilon_{0} n_{0} c\right)$.

Equation (3) describes the most general situation when the electrostatic potential in the crystal is induced by two distinct transport mechanisms: drift of charges in the biasing DC field plus the photogalvanic field and their diffusion. The relative strength of the diffusion and drift terms is determined by the dimensionless parameter

$$
\alpha=\frac{k_{\mathrm{B}} T}{e E_{\mathrm{ext}} l_{\perp}} .
$$

The diffusion contribution which leads to spatially asymmetric stimulated scattering dominates at large transverse wavenumbers of order $k_{\mathrm{D}}$. On the other hand the drift terms give the dominant contribution to the spatially symmetric MI which is prominent at much smaller transverse wavenumbers. Thus the term proportional to $\alpha$ in equation (3) is often neglected when studying MI.

The initial linear stage of the filamentation instability may be investigated by putting

$$
A(r, z)=A_{0} \mathrm{e}^{\mathrm{i} \beta z}\left(1+a \mathrm{e}^{\Gamma z+\mathrm{i} \mathbf{q} \cdot \mathbf{r}+\mathrm{i} \Omega t}+b \mathrm{e}^{\Gamma^{*} z-\mathrm{i} \mathbf{q} \cdot \mathbf{r}-\mathrm{i} \Omega t}\right)
$$

where $\Gamma$ represents the growth rate of the perturbation characterized by the transverse wavevector $\mathbf{q}$ and frequency detuning $\Omega$.

The steady state solution to equation (3) in the onedimensional plane wave limit with $\alpha=\xi=0$ is $\partial \phi / \partial x=$ $\left(1+E_{\mathrm{ph}} / E_{\mathrm{ext}}\right)\left|A_{0}\right|^{2} /\left(1+\left|A_{0}\right|^{2}\right)$ where $A_{0}$ is the amplitude of the plane wave which is assumed to vanish for $x \rightarrow \pm \infty$. It is therefore convenient to renormalize the potential as $\phi=$ $\left(1+E_{\mathrm{ph}} / E_{\mathrm{ext}}\right)\left|A_{0}\right|^{2} x /\left(1+\left|A_{0}\right|^{2}\right)+\tilde{\phi}$ so that the equations of motion become

$\frac{\partial A}{\partial z}-\mathrm{i} \nabla_{\perp}^{2} A=\mathrm{i}\left(\frac{\left(1+E_{\mathrm{ph}} / E_{\mathrm{ext}}\right)\left|A_{0}\right|^{2}}{1+\left|A_{0}\right|^{2}}+\frac{\partial \tilde{\phi}}{\partial x}\right) A$,

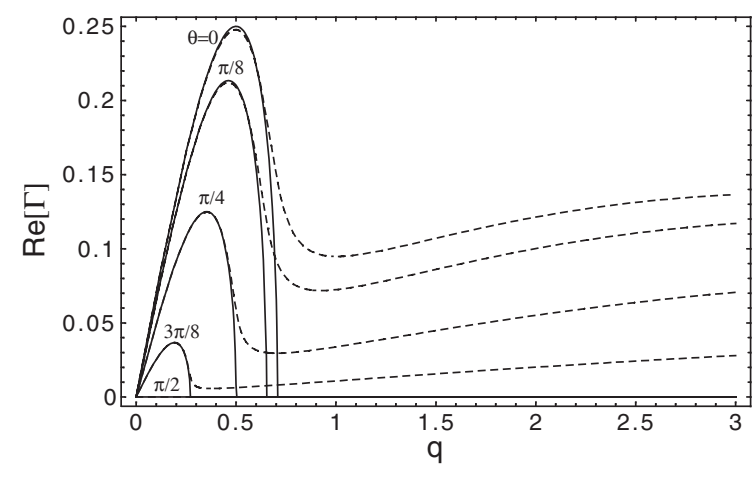

Figure 1. Growth rate as a function of spatial frequency $q$ for several values of the transverse angle $\theta$ for $A_{0}=1, \Omega=0, E_{\mathrm{ph}}=0$ and $\epsilon_{y} / \epsilon_{\mathrm{c}}=0.53$. The solid curves show the diffusionless case $(\alpha=\xi=0$ ) while the dashed curves show the effect of diffusion with $\alpha=0.046$ and $\xi=0.42$.

$$
\begin{aligned}
& \tau \frac{\partial}{\partial t}\left[\nabla_{\perp} \cdot\left(\hat{\epsilon}_{n} \nabla_{\perp} \tilde{\phi}\right)\right]+\nabla_{\perp}^{2} \tilde{\phi}+\nabla_{\perp} \tilde{\phi} \cdot \nabla_{\perp} \ln \bar{I} \\
& \quad=\frac{1-\left(E_{\mathrm{ph}} / E_{\mathrm{ext}}\right)\left|A_{0}\right|^{2}}{1+\left|A_{0}\right|^{2}} \frac{\partial}{\partial x} \ln \bar{I}+\frac{E_{\mathrm{ph}}}{E_{\mathrm{ext}} \bar{I}} \frac{\partial|A|^{2}}{\partial x}+\alpha \frac{\nabla_{\perp}^{2} \bar{I}}{\bar{I}}
\end{aligned}
$$

with $\bar{I}=\left(1+|A|^{2}\right) /\left[1-\xi \nabla_{\perp} \cdot\left(\hat{\epsilon}_{n} \nabla_{\perp} \tilde{\phi}\right)\right]$. The ansatz (4) then gives $\beta=\left(1+E_{\mathrm{ph}} / E_{\mathrm{ext}}\right)\left|A_{0}\right|^{2} /\left(1+\left|A_{0}\right|^{2}\right)$ which provides a continuous transition to the $1 \mathrm{D}$ solution.

Solving the linear problem defined by equations (4)-(6) gives the dispersion relation

$$
\begin{aligned}
\Gamma^{2}= & q^{2}\left[\frac{2\left|A_{0}\right|^{2}}{1+\left|A_{0}\right|^{2}}\left\{\frac{\cos ^{2}(\theta)}{1+\left|A_{0}\right|^{2}}\left(1+\frac{E_{\mathrm{ph}}}{E_{\mathrm{ext}}}\right)+\mathrm{i} \alpha q \cos (\theta)\right\}\right. \\
& \times\left\{1+\hat{\epsilon}_{n}(\theta)\left[\mathrm{i} \Omega \tau-\mathrm{i} \frac{q \cos (\theta) \xi}{1+\left|A_{0}\right|^{2}}\left(1-\left|A_{0}\right|^{2} \frac{E_{\mathrm{ph}}}{E_{\mathrm{ext}}}\right)\right.\right. \\
& \left.\left.\left.+\alpha \xi q^{2}\right]\right\}^{-1}-q^{2}\right]
\end{aligned}
$$

where $\hat{\epsilon}_{n}(\theta)=\cos ^{2}(\theta)+\frac{\epsilon_{y}}{\epsilon_{\mathrm{c}}} \sin ^{2}(\theta)$ with $\epsilon_{y}$ the static dielectric tensor component along $\hat{y}$ and $\theta$ the angle of $\mathbf{q}$ with respect to the $\hat{x}$ axis. In the limit of a single transverse dimension $\left(q_{y}=0\right)$ without diffusion, equation (7) reduces to the formula for the growth rate in saturable nonlinear media [6, 22].

The instability growth rate is given by $\operatorname{Re}[\Gamma]=$ $\sqrt{\operatorname{Re}\left[\Gamma^{2}\right]+\left|\Gamma^{2}\right|} / \sqrt{2}$. In figure 1 we show the growth rate as a function of the spatial frequency $q=|\mathbf{q}|$ for a few values of the angle $\theta$ using parameters characteristic of a photorefractive crystal as given in the appendix. The growth rate depends strongly on the angular orientation of the initial perturbation. In particular, it always attains the largest value when the wavevector of the perturbation coincides with the direction of the applied electric field $(\theta=0)$. As $\theta$ departs from zero the amplification of the perturbation decreases, and the growth rate becomes less strongly peaked at small $q$. The growth rate is an even function of $q$ provided that $\Omega=0$ and inspection of equation (7) shows that it falls of for large $q$ as $1 / q$. Interestingly equation (7) predicts there is no instability for perturbations with wavevectors perpendicular to the direction of the applied field.

We emphasize that the growth rate found from equation (7) is an even function of $\mathbf{q}$ also when there is a diffusional component of the nonlinear response. It is well known from studies of photorefractive two-wave mixing [25] that diffusion 
Two-dimensional modulational instability in photorefractive media
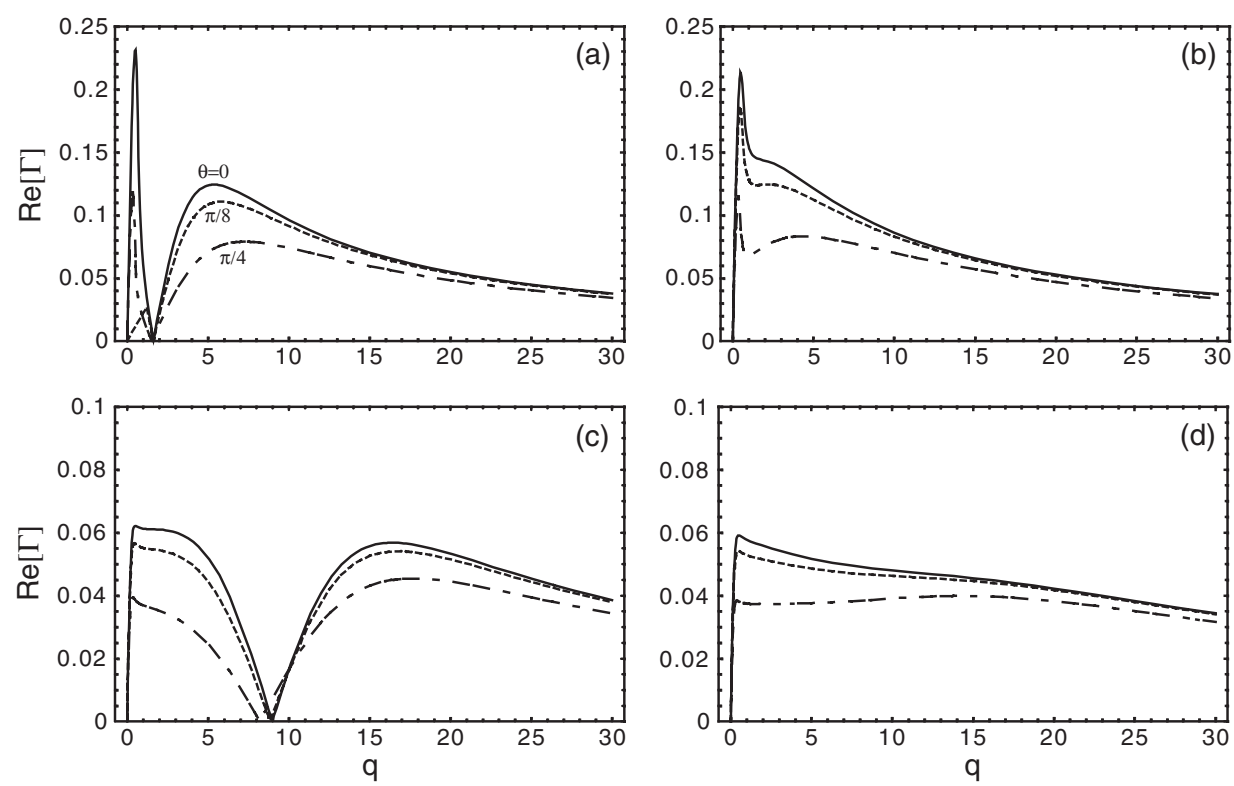

Figure 2. Growth rate of frequency shifted perturbations versus spatial frequency $q$ for several values of the transverse angle $\theta$ for $A_{0}=1$, $E_{\mathrm{ph}}=0, \epsilon_{y} / \epsilon_{\mathrm{c}}=0.53, \alpha=0.046$ and $\xi=0.42$. The subplots are for (a) $\Omega \tau=0.5$, (b) $\Omega \tau=-0.5$, (c) $\Omega \tau=4$ and (d) $\Omega \tau=-4$.

gives a nonlinear coupling coefficient proportional to $\mathbf{q} /\left(1+q^{2}\right)$ which is an odd function of $\mathbf{q}$. The apparent contradiction between the present results and those found in two-wave mixing stems from the fact that the perturbation ansatz of equation (4) includes terms proportional to $\mathrm{e}^{\mathrm{i} \mathbf{q} \cdot \mathbf{r}}$ and $\mathrm{e}^{-\mathrm{iq} \cdot \mathbf{r}}$. There is therefore no difference between the growth rates for positive and negative q. The two-wave mixing analysis which leads to an anti-symmetric gain spectrum assumes Bragg matching of the interacting waves, and therefore neglects the small Bragg mismatched component generated at $-\mathbf{q}$ in the presence of a seed beam at $+\mathbf{q}$. The linear analysis presented here includes the perturbations at $\pm \mathbf{q}$ but does not capture the asymmetry of the diffusional nonlinearity that occurs for thick gratings that are deep in the Bragg limit.

When the perturbation is frequency shifted with respect to the plane wave $(\Omega \neq 0$ ) the growth rate depends on the sign of $\Omega$. This is shown in figure 2 for $\Omega \tau= \pm 0.5$ and \pm 4 . For large $q$ the instability is dominated by diffusion, and is independent of the sign of $\Omega$, while for small $q$ the growth rate is slightly larger for positive $\Omega$. A curious feature of figure 2 is the existence of a zero growth rate at intermediate values of $q$ for positive $\Omega$. For $\theta=0$ and $E_{\mathrm{ph}}=0$ this occurs at

$$
\Omega_{\mathrm{c}} \tau=\left(1+\left|A_{0}\right|^{2}\right)\left[\frac{\xi q_{\mathrm{c}}}{\left(1+\left|A_{0}\right|^{2}\right)^{2}}+\alpha q_{\mathrm{c}}\left(1+\alpha \xi q_{\mathrm{c}}^{2}\right)\right] .
$$

The cancellation of the growth rate can be attributed to a destructive interference of the drift and diffusional parts of the nonlinearity. Additional analysis shows that cancellation occurs for negative $\Omega$ when $E_{\mathrm{ph}}$ is sufficiently large.

Finally, we note that the angular dependence takes on a simple form in the small $q$ limit where the diffusion contribution can be neglected. Putting $\alpha=\xi=0$ in (7) and assuming no frequency shift so $\Omega=0$ we obtain

$$
\operatorname{Re}[\Gamma]=q\left[\frac{2\left|A_{0}\right|^{2}}{\left(1+\left|A_{0}\right|^{2}\right)^{2}}\left(1+\frac{E_{\mathrm{ph}}}{E_{\mathrm{ext}}}\right) \cos ^{2} \theta-q^{2}\right]^{1 / 2} .
$$

This latter expression shows clearly the decline of the instability growth rate with increasing angle away from the $\hat{x}$ axis.

In order to verify the reliability of the linear approximation discussed above we resorted to numerical analysis of the full two-dimensional model governing propagation of optical beams in a photorefractive medium: equations (2), (3). For simplicity and comparison with the experimental conditions discussed below we only considered the frequency degenerate $(\Omega=0)$ and short Debye length $(\alpha=\xi=0)$ limit. We also assumed the lack of a photogalvanic effect $\left(E_{\mathrm{ph}}=0\right)$. We used a split step fast Fourier transform code to solve the propagation equation (2) and a finite difference technique to find the electrostatic potential and refractive index change induced by the beam. Results of these calculations are shown in figures 3-5. In all cases the direction of the applied DC field is horizontal (along the $x$ axis). In figure 3 we show a few examples of numerical simulations depicting results of propagation of the wide Gaussian beam with initial random perturbation of its amplitude, for a few values of the beam peak intensity. These graphs illustrate the inherently anisotropic nature of the instability. Initially random perturbations lead to amplification of the perturbation with almost zero $y$ component of the wavevector. This leads to the appearance of beam modulation in the form of roughly vertically oriented stripes. Graphs in the bottom row display the spatial spectrum (a zero-frequency component has been removed for clarity of presentation) of the resulting intensity distribution. Notice that the spatial frequency with the highest growth rate varies with the peak intensity of the beam, which is in agreement with the prediction of equation (9). For comparison figure 4 shows the intensity distribution obtained with the Gaussian beam propagating in a standard isotropic saturable medium. The beam experiences modulational instability but this time all amplified spatial frequencies are located on a ring, reflecting the isotropic nature of the nonlinear process. 


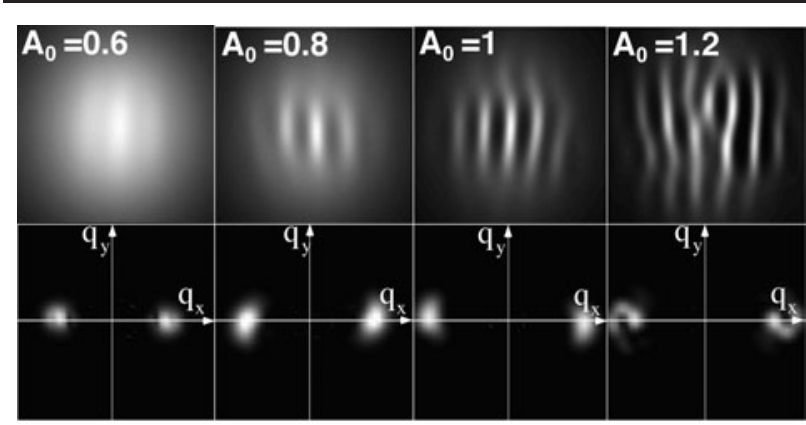

Figure 3. The intensity distribution (top) and its spatial spectrum (bottom) for a Gaussian beam with initial random noise, after propagation over a distance of $5 \mathrm{~mm}$ in a photorefractive crystal. The size of the computational window is $200 \mu \mathrm{m} \times 200 \mu \mathrm{m}$. A zero-frequency component has been removed from the spectrum.

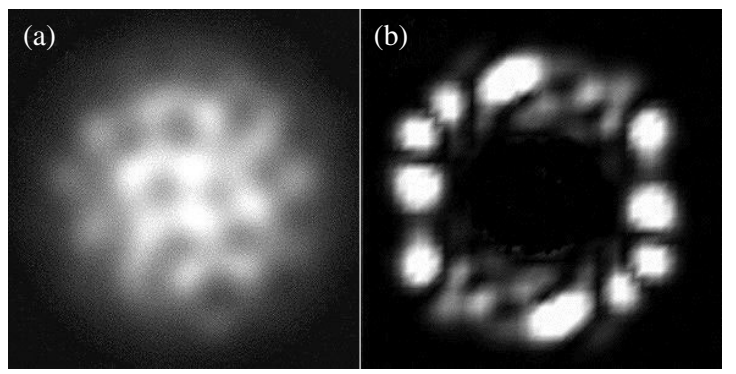

Figure 4. The intensity distribution (a) and its spatial spectrum (b) for a broad Gaussian beam with a superimposed initial random noise, after propagation over a distance of $5 \mathrm{~mm}$ in an isotropic nonlinear medium. For clarity of presentation the zero-frequency component has been removed from the spectrum.

The complete absence of instability for $\theta=\pi / 2$ and its apparent one-dimensional character as depicted in figure 3 are a direct consequence of the anisotropy of the nonlinear response of the photorefractive medium. The light induced focusing power is roughly three times stronger in the direction of the applied DC field than in the direction perpendicular to it [15]. Unlike in the isotropic system where all spatial frequencies corresponding to highest growth rate are amplified (figure 4), in the photorefractive crystal the highest gain is experienced only by perturbations with $q_{y} \approx 0(\theta \approx 0)$. Therefore only these frequencies will contribute to the initial stages of the modulational instability described by the linear theory. Spatial perturbations with nonzero $q_{y}$ components have much weaker growth rates and will play an important role only after the $1 \mathrm{D}$ structure with $q_{y} \approx 0$ has reached sufficiently high intensity [18]. Then the full two-dimensional break-up and subsequent filamentation of the beam will follow [23, 26]. However, the full analysis of such a process is beyond the scope of the present paper.

Next we simulated propagation of a broad Gaussian beam with its amplitude perturbed by a spatially periodic modulation. The angle $\theta$ which determines the angular orientation of the perturbation with respect to the direction of the applied DC field was varied from $\theta=0^{\circ}$ to $90^{\circ}$. The strength of the perturbation (relative to the peak intensity of the beam) was less than $10^{-2}$. Results for the propagation of this beam over a distance of five millimetres are shown in figure 5. Each row of this figure corresponds to a different spatial frequency of

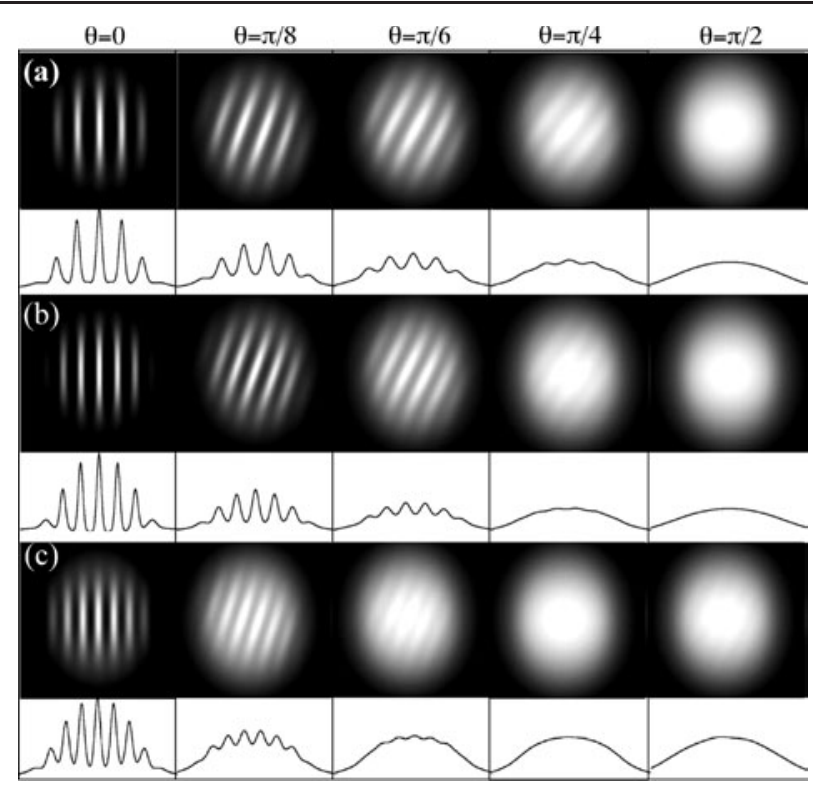

Figure 5. The intensity distribution of the optical beam in a biased photorefractive crystal with an initially imposed periodic perturbation as a function of the angular orientation of the perturbation and its wavevector $(q)$. The peak intensity $A_{0}^{2}=1$; the DC biasing electric field is applied along the horizontal $(x)$ direction. The size of the computational window is $200 \mu \mathrm{m} \times 200 \mu \mathrm{m}$.

the initial perturbation. A decrease in the amplification of the perturbation with increasing angle $\theta$ is evident.

To verify our theoretical findings we conducted experiments using a crystal of photorefractive strontium barium niobate as the nonlinear medium. The experimental set-up is analogous to that used in our earlier studies of photorefractive soliton formation [13]. The crystal was $5 \times$ $5 \times 10 \mathrm{~mm}^{3}$ in size with the optical beam propagating along the $10 \mathrm{~mm}$ axis and a DC electric field of $1.1 \mathrm{kV}$ applied along the $5 \mathrm{~mm}$ long $\hat{c}$ axis. The optical beam $(1 \mathrm{~mW})$ from a solid state laser $(\lambda=532 \mathrm{~nm})$ was loosely focused at the input face of the crystal. The output intensity distribution was imaged by a CCD camera and stored in a computer. The crystal was illuminated by a broad white light beam which was used to control the degree of saturation. Typically, the peak intensity of the incident beam was of the same order as the average intensity of the white light background. We used either an unperturbed beam or a beam with superimposed weak periodic perturbations. Results of the experiments are shown in figures 6 and 7. Figure 6(a) shows the light intensity distribution at the exit facet of the crystal (after $10 \mathrm{~mm}$ of propagation) in the case where the incoming beam was not intentionally perturbed. It is evident that nonlinearity induces modulational instability which leads to the formation of quasione-dimensional vertical stripes oriented perpendicularly to the direction of the applied DC field. Figure 6(b) depicts the corresponding Fourier spectrum of the outgoing beam where the two distinct peaks with almost zero $q_{y}$ components clearly indicate the anisotropic character of the instability. The presence of the small $y$ component in the spectrum is the result of a slight misalignment of the crystal.

Finally we investigated the role of anisotropy in the modulational instability of a broad beam with an initially imposed periodic perturbation. To this end the incoming 
Two-dimensional modulational instability in photorefractive media

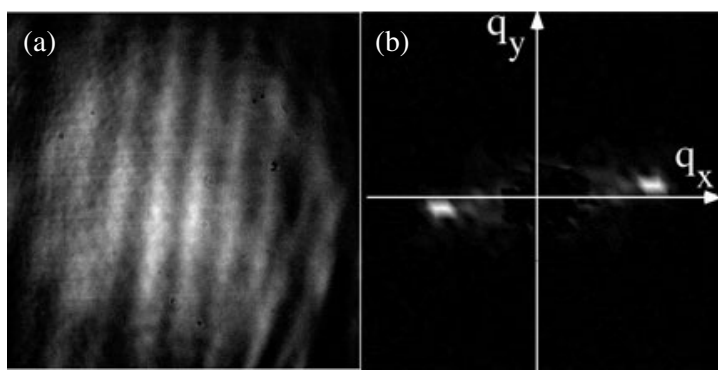

Figure 6. Experimentally observed self-induced modulational instability of the initially Gaussian beam (width $=200 \mu$ ). (a) The light intensity distribution; (b) the spatial spectrum (with the zero-frequency component removed). The amplitude of the external biasing DC field $E_{\text {ext }}=3 \mathrm{kV} \mathrm{cm}^{-1}$. The DC field is applied in the horizontal direction. The width of the experimental window is $320 \mu \mathrm{m} \times 320 \mu \mathrm{m}$

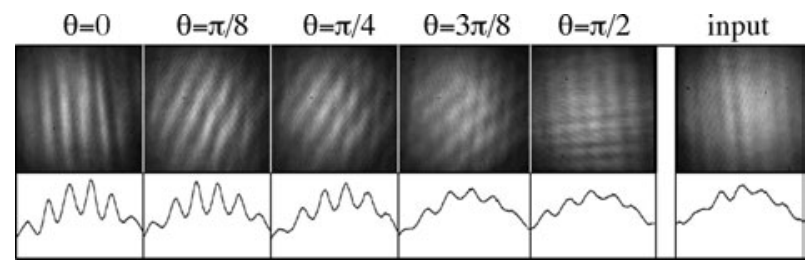

Figure 7. The experimentally observed dependence of the modulational instability of the broad Gaussian beam on the angular orientation of the initial periodic perturbation. The amplitude of the external DC electric field $E_{\text {ext }}=2.2 \mathrm{kV} \mathrm{cm}^{-1}$, with the field applied along the horizontal direction.

Gaussian beam was initially transmitted through a parallel plate which resulted in the appearance of a weak spatial periodic modulation of the beam wavefront. By rotating the plate we were able to change the orientation of this modulation. The perturbed beam subsequently propagated through the biased photorefractive crystal. Results of this experiment are shown in figure 7. Greyscale plots in the top row of this figure represent the light intensity distribution at the output face of the photorefractive crystal corresponding to different angular orientations of the periodic pattern characterized by the angle $(\theta)$. Graphs in the bottom row illustrate the corresponding intensity profile. As figure 7 clearly shows, the amplification of the perturbation decreases rapidly as the angle departs from $\theta=0$. The rightmost plot shows the intensity pattern at the input face of the crystal. For better visualization we plot in figure 8 the experimentally measured growth rate (normalized to its maximum value) as a function of the angle $\theta$. The points represent experimental data while the line is a theoretical fit (equation (9)) with $E_{\mathrm{ph}}=0, A_{0}=2.6, \xi=0.42, \alpha=0$ and $q=0.35$. Again, the drop in amplification of the perturbation for increasing $\theta$ is evident.

In conclusion, we have investigated modulational instability of plane waves and finite beams in photorefractive nonlinear media biased with a DC electric field. We showed that the growth rate of the perturbation is affected by the inherent anisotropy of the nonlinear response. It is highest for perturbations whose wavevectors correspond to the direction of the biasing DC field. For arbitrarily oriented perturbations the effect of the anisotropy manifests itself in a decrease of the effective strength of the nonlinear response until it reaches zero for wavevectors perpendicular to the direction of the field.

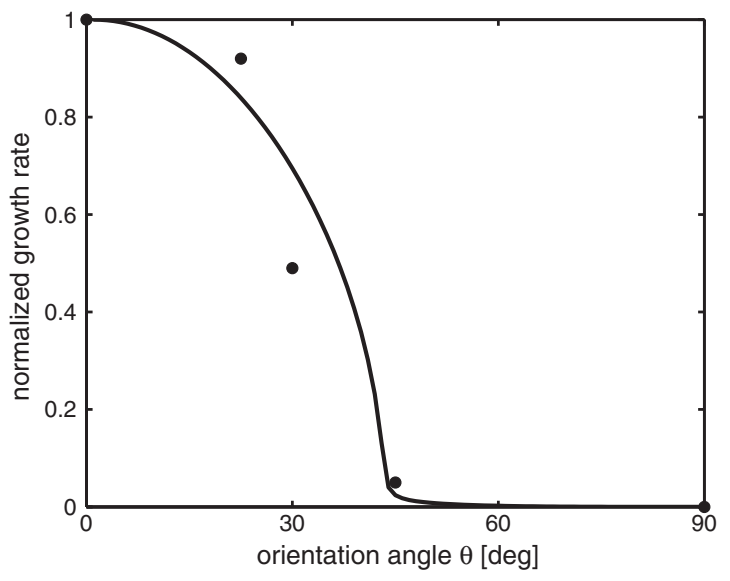

Figure 8. The experimentally measured normalized growth rate of an initial periodic perturbation imposed onto the broad Gaussian beam as a function of the angular orientation of the initial pattern Dots - experimental points; curve - theoretical fit (formula (9)).

Our theoretical predictions were confirmed by experimental observations on strontium barium niobate crystals.

\section{Acknowledgment}

The work of WK and GM was supported by the Australian Research Council.

\section{Appendix. Derivation of equations}

The set of equations describing the optical properties of a photorefractive crystal, known as the Kukhtarev equations [25], are

$$
\begin{gathered}
\frac{\partial N_{\mathrm{D}}^{+}}{\partial t}=\left(\beta+\sigma I_{\mathrm{em}}\right)\left(N_{\mathrm{D}}-N_{\mathrm{D}}^{+}\right)-\gamma_{\mathrm{r}} n_{\mathrm{e}} N_{\mathrm{D}}^{+}, \\
\rho=e\left(N_{\mathrm{D}}^{+}-N_{\mathrm{A}}-n_{\mathrm{e}}\right), \\
\mathbf{J}=e \mu n_{\mathrm{e}} \mathbf{E}+\mu k_{\mathrm{B}} T \nabla n_{\mathrm{e}}+\beta_{\mathrm{ph}}\left(N_{\mathrm{D}}-N_{\mathrm{D}}^{+}\right) I_{\mathrm{em}} \hat{c}, \\
\nabla \cdot\left(\epsilon_{0} \hat{\epsilon} \mathbf{E}\right)=\rho, \\
\frac{\partial \rho}{\partial t}+\nabla \cdot \mathbf{J}=0 .
\end{gathered}
$$

Here $N_{\mathrm{D}}, N_{\mathrm{D}}^{+}, N_{\mathrm{A}}$ and $n_{\mathrm{e}}$ are the densities of donors, ionized donors, acceptors and conduction electrons, $\beta$ and $\sigma$ are the coefficients of thermal excitation and photoexcitation, $I_{\mathrm{em}}$ is the optical intensity, $\gamma_{\mathrm{r}}$ is the electron recombination coefficient, $-e$ is the charge on an electron, $\epsilon_{0}$ is the permeability of vacuum, $\hat{\epsilon}$ is the static dielectric tensor, $k_{\mathrm{B}}$ is the Boltzmann constant, $T$ the temperature, $\mu$ the electron mobility, $\rho$ the charge density, $\mathbf{J}$ the current and $\mathbf{E}$ the static electric field. Note that the coefficient $\sigma$ includes the photogalvanic contribution due to $\beta_{\text {ph }}$ so we can write the total photoexcitation coefficient as $\sigma=\sigma_{1}+\beta_{\mathrm{ph}}$.

We analyse these equations following the approach of [14]. In the absence of thermal excitation or photoexcitation $n_{\mathrm{e}}=0$, so the condition $\left\langle N_{\mathrm{D}}^{+}\right\rangle=\left\langle N_{\mathrm{A}}\right\rangle$, where \langle\rangle denotes a spatial average, ensures bulk charge neutrality. The negatively charged acceptors do not participate in the photoexcitation dynamics, so the density $N_{\mathrm{A}}$ is fixed and serves to limit the 
magnitude of the photoexcited space charge field. To analyse the Kukhtarev equations we assume $N_{\mathrm{D}} \gg N_{\mathrm{A}} \gg n_{\mathrm{e}}$. Gauss's law (A.1d) then gives

$$
N \equiv \frac{N_{\mathrm{D}}^{+}}{N_{\mathrm{A}}}=1+\frac{1}{e N_{\mathrm{A}}} \nabla \cdot \epsilon_{0} \hat{\epsilon} \mathbf{E}
$$

Introducing the Debye wavenumber for charge motion along the $\hat{c}$ axis as $k_{\mathrm{D}}=e \sqrt{N_{\mathrm{A}} /\left(k_{\mathrm{B}} T \epsilon_{0} \epsilon_{\mathrm{c}}\right)}$, where $\epsilon_{\mathrm{c}}$ is the component of the dielectric tensor along $\hat{c}$ and the characteristic field $\tilde{E}=k_{\mathrm{B}} T k_{\mathrm{D}} / e=e N_{\mathrm{A}} /\left(\epsilon_{0} \epsilon_{\mathrm{c}} k_{\mathrm{D}}\right)$, we can write the last expression as

$$
N=1+\frac{1}{k_{\mathrm{D}} \tilde{E}} \nabla \cdot \hat{\epsilon}_{n} \mathbf{E}
$$

with $\epsilon_{n}$ the static dielectric tensor divided by $\epsilon_{\mathrm{c}}$.

The assumption of fast carrier recombination implies that $\partial n_{\mathrm{e}} / \partial t$ can be set to zero in the equation for charge continuity. It follows that

$$
\begin{aligned}
\frac{\partial M}{\partial t} & +\frac{1}{\epsilon_{0} \epsilon_{\mathrm{c}} k_{\mathrm{D}} \tilde{E}} \nabla \cdot\left[e \mu n_{\mathrm{e}} \mathbf{E}+\mu k_{\mathrm{B}} T \nabla n_{\mathrm{e}}\right. \\
& \left.+\beta_{\mathrm{ph}}\left(N_{\mathrm{D}}-N_{\mathrm{D}}^{+}\right) I_{\mathrm{em}} \hat{c}\right]=0
\end{aligned}
$$

where $M=N-1$. To proceed we use equation (A.1a) to write

$$
n_{\mathrm{e}}=-\frac{1}{\gamma_{\mathrm{r}} N} \frac{\partial N}{\partial t}+\frac{\beta}{\gamma_{\mathrm{r}}}\left(1+I_{\mathrm{em}} / I_{\mathrm{s}}\right) \frac{N_{\mathrm{D}} / N_{\mathrm{A}}-N}{N}
$$

where $I_{\mathrm{s}}=\beta / \sigma$ is the saturation intensity for which the rate of thermal excitation equals the rate of photoexcitation. With fast carrier recombination and $N_{\mathrm{D}} \gg N_{\mathrm{A}}$ we have

$$
n_{\mathrm{e}} \simeq \frac{\beta}{\gamma_{\mathrm{r}} N}(1+I) \frac{N_{\mathrm{D}}}{N_{\mathrm{A}}}
$$

with $I=I_{\mathrm{em}} / I_{\mathrm{s}}$. The characteristic density of electrons associated with $I_{\mathrm{S}}$ is $n_{0}=\left(\beta / \gamma_{\mathrm{r}}\right)\left(N_{\mathrm{D}} / N_{\mathrm{A}}\right)$ so the electron density can be written as

$$
n_{\mathrm{e}}=n_{0} \frac{1+I}{1+M} .
$$

Furthermore the photogalvanic term can be written as

$$
\begin{aligned}
\beta_{\mathrm{ph}}\left(N_{\mathrm{D}}-N_{\mathrm{D}}^{+}\right) I_{\mathrm{em}} & \simeq \beta_{\mathrm{ph}} N_{\mathrm{D}} I_{\mathrm{em}} \\
& =\beta_{\mathrm{ph}} \frac{N_{\mathrm{A}} n_{\mathrm{e}} \gamma_{\mathrm{r}} N}{\sigma} \frac{I}{1+I} \\
& =\beta_{\mathrm{ph}} \frac{N_{\mathrm{A}} n_{0} \gamma_{\mathrm{r}}}{\sigma} I .
\end{aligned}
$$

Defining the characteristic relaxation time of the electric field $t_{0}=\frac{\epsilon_{0} \epsilon_{\mathrm{c}}}{e \mu n_{0}}$ and the photogalvanic field $E_{\mathrm{ph}}=\frac{\beta_{\mathrm{ph}} \gamma_{\mathrm{r}} N_{\mathrm{A}}}{e \mu \sigma}$, equation (A.2) can be written as

$$
t_{0} \frac{\partial M}{\partial t}+\frac{1}{k_{\mathrm{D}} \tilde{E}} \nabla \cdot\left[\frac{1+I}{1+M} \mathbf{E}+\frac{\tilde{E}}{k_{\mathrm{D}}} \nabla \frac{1+I}{1+M}+E_{\mathrm{ph}} I \hat{c}\right]=0 .
$$

This equation coincides with [14], equation (2), with $\chi=0$ and $\delta=0$.

We are interested in the situation where the optical beam is small compared to the size of the nonlinear medium. The externally applied bias field is $E_{\text {ext }}=V / L_{x}$ with $V$ the applied voltage and $L_{x}$ the width of the medium along $\hat{x}$ which is taken to coincide with the $\hat{c}$ axis. It is convenient to subtract this field from the optically induced field so that the field $\mathbf{E}_{\mathrm{r}}=\mathbf{E}-E_{\mathrm{ext}} \hat{x}$ vanishes at the boundaries of the medium. Using $\mathbf{E}_{\mathrm{r}}$ instead of $\mathbf{E}$ in equation (1) results in only a small change in the wavenumber of the beam which has no physical importance for this work. We then introduce a potential through the relation $\mathbf{E}_{\mathrm{r}}=-\tilde{E} \nabla_{\perp} \phi$ so that equation (A.5) can be written as

$$
\begin{gathered}
t_{0} \frac{\partial}{\partial t}\left[\nabla \cdot\left(\hat{\epsilon}_{n} \nabla \phi\right)\right]+\frac{1+I}{1+M} \nabla^{2} \phi+\nabla \phi \cdot \nabla \frac{1+I}{1+M} \\
=\frac{E_{\mathrm{ext}}}{\tilde{E}} \frac{\partial}{\partial x} \frac{1+I}{1+M}+\frac{E_{\mathrm{ph}}}{\tilde{E}} \frac{\partial I}{\partial x}+\frac{1}{k_{\mathrm{D}}} \nabla^{2} \frac{1+I}{1+M} .
\end{gathered}
$$

In the situation of interest here where the optical field is linearly polarized along $\hat{x}$, the nonlinear increment to the refractive index is given by $n_{2}(r, z)=-\frac{1}{2} n_{0}^{3} r_{33} E_{x}(r, z)$, with $r_{33}$ the relevant component of the electro-optic tensor and $E_{x}$ the $\hat{x}$ component of the low frequency electric field in the medium. In a paraxial approximation the optical field therefore satisfies the parabolic equation

$$
\frac{\partial A}{\partial z}-\frac{\mathrm{i}}{2 k} \nabla_{\perp}^{2} A=\mathrm{i}\left(\frac{k}{2} n_{0}^{2} r_{33} \tilde{E}\right) \frac{\partial \phi}{\partial x} A(r, z) .
$$

Within the same paraxial approximation we drop the longitudinal derivatives in equation (A.6) so that the gradient operator becomes $\nabla_{\perp}=\hat{x} \partial / \partial x+\hat{y} \partial / \partial y$. Finally, introducing the spatial scales $l_{\perp}$ and $l_{\|}$and redefining the coordinates and variables through $(x, y) / l_{\perp} \rightarrow(x, y), z / l_{\|} \rightarrow z$, $\phi\left(\tilde{E} / E_{\text {ext }}\right)\left(1 / l_{\perp}\right) \rightarrow \phi$, gives the normalized set

$\frac{\partial A}{\partial z}-\mathrm{i} \nabla_{\perp}^{2} A=\mathrm{i} \frac{\partial \phi}{\partial x} A$,

$\tau \frac{\partial}{\partial t}\left[\nabla_{\perp} \cdot\left(\hat{\epsilon}_{n} \nabla_{\perp} \phi\right)\right]+\nabla_{\perp}^{2} \phi+\nabla_{\perp} \phi \cdot \nabla_{\perp} \ln \bar{I}$

$=\frac{\partial}{\partial x} \ln \bar{I}+\frac{E_{\mathrm{ph}}}{E_{\mathrm{ext}} \bar{I}} \frac{\partial I}{\partial x}+\frac{k_{\mathrm{B}} T}{e E_{\mathrm{ext}} l_{\perp}} \frac{\nabla_{\perp}^{2} \bar{I}}{\bar{I}}$

where $\tau=t_{0} / \bar{I}, \bar{I}=(1+I) /\left[1-\xi \nabla_{\perp} \cdot\left(\hat{\epsilon}_{n} \nabla_{\perp} \phi\right)\right], \xi=$ $E_{\text {ext }} /\left(\tilde{E} l_{\perp} k_{\mathrm{D}}\right), l_{\|}=2 /\left(k n_{0}^{2} r_{33} E_{\text {ext }}\right)$ and $l_{\perp}=\sqrt{l_{\|} /(2 k)}$.

We can calculate characteristic values for the theoretical parameters for the SBN crystal used in the experimental work. Using data from [27] we have $\epsilon_{\mathrm{c}}=880, \epsilon_{y}=470$ and $N_{\mathrm{A}} \simeq 10^{16} \mathrm{~cm}^{-3}$ so the Debye length is $2 \pi / k_{\mathrm{D}}=2.2 \mu \mathrm{m}$, and the characteristic internal field is $\tilde{E}=730 \mathrm{~V} \mathrm{~cm}^{-1}$. The optical parameters are $n_{0}=2.3, \lambda=0.532 \mu \mathrm{m}$, while the electro-optic coefficient of our crystal was measured to be $r_{33} \sim 180 \mathrm{pm} \mathrm{V}^{-1}$. With a typical applied field of $E_{\text {ext }}=2.2 \mathrm{kV} \mathrm{cm}^{-1}$ we have $l_{\|}=350 \mu \mathrm{m}, l_{\perp}=2.5 \mu \mathrm{m}$, $\alpha=0.046$ and $\xi=0.42$.

\section{References}

[1] Bespalov V I and Talanov V I 1966 Filamentary structure of light beams in nonlinear liquids Pis. Zh. Eksp. Teor. Fiz. 3 471-6

Bespalov V I and Talanov V I 1966 JETP Lett. 3 307-10 (Engl. Transl.)

[2] Dyachenko S, Newell A C, Pushkarev A and Zakharov V E 1992 Optical turbulence: weak turbulence, condensates and collapsing filaments in the nonlinear Schrödinger equation Physica D 57 96-160

[3] Trillo S and Ferro P 1995 Modulational instability in second-harmonic generation Opt. Lett. 20438 
Two-dimensional modulational instability in photorefractive media

He H, Drummond P D and Malomed B A 1996 Modulational stability in dispersive optical systems with cascaded nonlinearity Opt. Commun. $\mathbf{1 2 3} 394$

Musslimani Z H and Malomed B A 1998 Modulational instability in bulk dispersive quadratically nonlinear media Physica D 123 235-43

[4] Wyller J, Krolikowski W, Bang O and Rasmussen J J 2002 Generic features of modulational instability in nonlocal Kerr media Phys. Rev. E 66066615

[5] Peccianti M, Conti C and Assanto G 2003 Optical modulational instability in a nonlocal medium Phys. Rev. E $68025602(\mathrm{R})$

[6] Shih M-F, Jeng C-C, Sheu F-W and Lin C-Y 2002 Spatiotemporal optical modulation instability of coherent light in noninstantaneous nonlinear media Phys. Rev. Lett. 88133902

[7] Kip D, Soljacic M, Segev M, Eugenieva E and Christodoulides D N 2000 Modulation instability and pattern formation in spatially incoherent light beams Science 290495

[8] Benjamin T B and Feir J E 1967 J. Fluid Mech. 27417

[9] Hasegawa A 1975 Plasma Instabilities and Nonlinear Effects (Heidelberg: Springer)

[10] Strecker K E, Partridge G B, Truscott A G and Hulet R G 2002 Formation and propagation of matter wave soliton trains Nature 417150

Carr L D and Brand J 2004 Spontaneous soliton formation and modulational instability in Bose-Einstein condensates Phys. Rev. Lett. 92040401

[11] Iturbe Castillo M D, Marquez-Aguilar P A, Sanchez-Mondragon J, Stepanov S and Vysloukh V 1994 Spatial solitons in photorefractive $\mathrm{Bi}_{12} \mathrm{TiO}_{20}$ with drift mechanism of nonlinearity Appl. Phys. Lett. 64408

[12] Segev M, Crosignani B, DiPorto P, Valley G C and Yariv A 1994 Steady-state spatial screening solitons in photorefractive materials with external applied field Phys. Rev. Lett. 733211

[13] Krolikowski W, Luther-Davies B and Denz C 2003 Photorefractive solitons IEEE J. Quantum Electron. 393

[14] Zozulya A A and Anderson D Z 1995 Propagation of an optical beam in a photorefractive medium in the presence of a photogalvanic nonlinearity or an externally applied electric field Phys. Rev. A 511520
[15] Korneev N, Marquez Aguilar P A, Sanchez Mondragon J J, Stepanov S, Klein M and Wechsler B 1996 Anisotropy of steady-state two-dimensional lenses in photorefractive crystals with drift nonlinearity J. Mod. Opt. 43311

[16] Zozulya A A, Anderson D Z, Mamaev A V and Saffman M 1996 Self-focusing and soliton formation in media with anisotropic nonlocal material response Europhys. Lett. 36419

[17] Krolikowski W, Saffman M, Luther-Davies B and Denz C 1998 Anomalous interaction of spatial solitons in photorefractive media Phys. Rev. Lett. 80 3240-3

[18] Mamaev A V and Saffman M 1996 Hexagonal optical patterns in anisotropic nonlinear media Europhys. Lett. 34669

[19] Carvalho M I, Singh S R and Christodoulides D N 1996 Modulational instability of quasi-plane-wave optical beams biased in photorefractive crystals Opt. Commun. 126167

[20] Iturbe-Castillo M D, Torres-Cisneros M, Sánchez-Mondragón J J, Chávez-Cerda S, Stepanov S I, Vysloukh V A and Torres-Cisneros G E 1995 Experimental evidence of modulation instability in a photorefractive $\mathrm{Bi}_{12} \mathrm{TiO}_{20}$ crystal Opt. Lett. 201853

[21] Apolinar-Iribe A, Korneev N, Vysloukh V and Gómez-Sarabia C M 2002 Transverse modulational instability of periodic light patterns in photorefractive strontium barium niobate crystal Opt. Lett. 272088

[22] Kivshar Y S, Anderson D and Lisak M 1993 Modulational instabilities and dark solitons in a generalized nonlinear Schrödinger equation Phys. Scr. 47 679-81

[23] Mamaev A V, Saffman M, Anderson D Z and Zozulya A A 1996 Propagation of light beams in anisotropic nonlinear media: from symmetry breaking to spatial turbulence Phys. Rev. A 54 870-9

[24] Zozulya A A, Anderson D Z, Mamaev A V and Saffman M 1998 Solitary attractors and low-order filamentation in anisotropic self-focusing media Phys. Rev. A 57 522-34

[25] Kukhtarev N V, Markov V B, Odulov S G, Soskin M S and Vinetskii V L 1979 Ferroelectrics 22949

[26] Infeld E and Lenkowska-Czerwińska T 1997 Analysis of stability of light beams in nonlinear photorefractive media Phys. Rev. E 55 6101-6

[27] Ewbank M D, Neurgaonkar R R, Cory W K and Feinberg J 1987 Photorefractive properties of strontium barium niobate J. Appl. Phys. 62374 